\title{
Modelling the Accessibility of Adult Psychology Services Using Discrete Event Simulation
}

\author{
Matthew Howells \\ School of Mathematics \\ Cardiff University \\ HowellsMA@cardiff.ac.uk
}

\author{
Liz Andrew \\ Adult Psychology \\ St. Cadoc's Hospital \\ Liz.Andrew@wales.nhs.uk
}

\author{
Daniel Gartner \\ School of Mathematics \\ Cardiff University \\ GartnerD@ cardiff.ac.uk
}

\begin{abstract}
With a growing number of people seeking treatment for mental health problems, mental health services are consequently coming under increased pressure resulting in longer waiting times and worsening of mental health problems. Service underfunding, overworked staff, and the looming threat of further demand due to the COVID-19 pandemic only add to the concerns. Hence it is imperative the efficiencies of these services are maximised to allow better access to quality treatment. We created a Discrete Event Simulation model to replicate the current clinical approach taken in an adult psychology clinic in the U.K.'s National Health Service. The model identifies bottlenecks in the service, and provides results on how different staffing scenarios could alleviate challenges.
\end{abstract}

\section{Introduction}

Mental health is an issue of growing concern around the world, with more and more people reporting experiencing, or having experienced mental health issues in their lives. The consequence of this increased demand is that mental health services come under increased pressure, and due to problems, such as underfunding and low staffing levels, these services are often ill-equipped to effectively treat patients. In such cases, this results in long waiting times for the patients' assessments and interventions. In the long term, this can exacerbate their mental health problems, resulting in further deterioration of their health, as well as increased long-term costs to health services and the wider economy. Thus, it is vital that the efficiencies and accessibility of these services are maximised, in order to grant more people access to quality mental health treatment, as well as easing the burden on the staff in these services.

Operational research presents us with an arsenal of powerful techniques to model healthcare services such as primary care mental health services [1].
Among these methods is simulation, and in particular, Discrete Event Simulation (DES), which allows us to create mathematical models of services through discrete sequences of events in time. In doing so, we can carry out what-if analyses of different scenarios and configurations of the services in a safely and cost-effective manner. Thus, it is a particularly useful tool for healthcare settings, and in turn, mental healthcare settings.

This paper presents a DES model of an adult psychology clinic in the U.K., which is used to explore the impact of different staffing configurations on reducing patient waiting times in the service. Doing so also aims to highlight the practicality of DES in helping to improve the efficiencies of mental health services.

This research was undertaken in accordance with the Aneurin Bevan University Health Board (ABUHB), a part of NHS Wales. The health board reports experiencing prolonged waiting times for initial assessments in its adult psychology clinics. The health board was particularly interested in whether a DES model could answer the research question of to what extent a dedicated care coordination role can improve efficiencies in mental health services. Patients in secondary care mental health services are required by Welsh law to have a Care Coordinator to aid them through their treatment plan. Currently, the psychologists in these services must undertake the Care Coordinator roles alongside their therapy duties. This results in the staff being overburdened with work, and as a further consequence, this leaves staff often unavailable to carry out frequent assessments of patients entering the service, which results in the increasing waiting times.

Our DES model aims to assess the effect employing dedicated staff to take over the Care Coordinator roles from the psychologists has on improving patient flow through the service.

The remainder of this paper is structured as follows. Section 2 will provide some background and highlight related work in the field of Simulation Modelling applied to Mental Health Services followed by an 
overview of our simulation model in Section 3. In Section 4, we will present our findings broken down by different metrics and level of detail. Section 5 will summarise the limitations of our modelling approach and includes discussions, followed by concluding remarks in Section 6.

\section{Background and Related Work}

\subsection{Background}

Mental health problems are a prevalent issue in many industrial countries. According to the Welsh Health Survey 2015, it is estimated that $13 \%$ of the adult population were receiving treatment for a mental illness, a figure that had steadily increased annually from approximately $9 \%$ in 2008 [2].

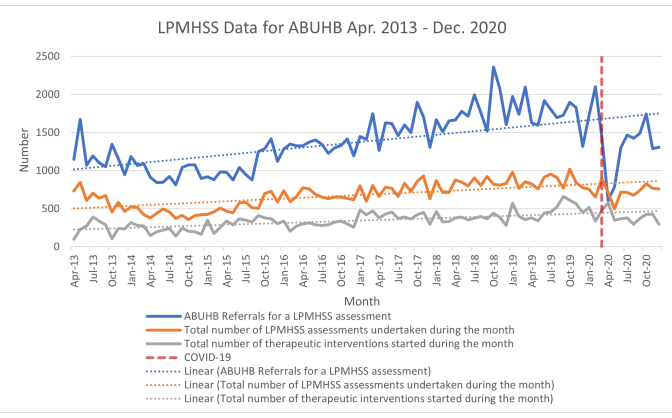

Figure 1. LPCMHSS Data for Aneurin Bevan University Health Board from April 2013 - December 2020. Data from StatsWales [3]

Through analysing the figures on monthly referrals to Local Primary Care Mental Health Support Services (LPCMHSS) for ABUHB on the StatsWales website [3], we can see from Figure 1 that there has been an increasing trend in the number of referrals to LPCMHSS over the period of April 2013 to December 2020. Yet over this period, except for April 2020 due to the introduction of first U.K. national COVID-19 lockdown in March 2020, the number of monthly referrals has consistently been higher than the number of monthly LPCMHSS assessments undertaken in the same month. This is also with the trend in referrals increasing at a higher rate than the increase in monthly assessments too. Similar trends are viewed across Wales as a whole, giving the indication that despite a growing number of people being referred for primary care services, there is not a noteworthy increase in the accessibility of these services to match it.

The Royal College of Psychiatrists 2019 Census highlights that $12.7 \%$ of consultant psychiatrist roles in Wales are vacant, which is the highest figure amongst the four UK nations, highlighting the lower staffing levels seen in the service [4].

\subsection{Relevant Work}

Operational research techniques have been employed as useful tools in a myriad of healthcare settings, the extent to which has been well documented in literature [5, 6]. Simulation models are useful in healthcare settings in dealing with system design and planning issues including improving patient flow and improving resource allocations and efficiencies, by allowing us to test different configurations and scenarios in a safe and cost-effective way.

Despite this, it has been noted that there is a scarcity of literature reviews of the applications of simulation modelling to mental healthcare and services, as evidenced by Long and Meadows (2018) [7]. In turn, this holds true for DES as well, and Noorain et al. (2019) [8] found just ten applications of DES to mental health services over the period from 2000 to 2019.

Our searching of the Scopus, Web of Sciences, and PubMed databases identified a further nine papers to Noorain et al. (2019) [8], giving a total of nineteen applications of DES to mental health services. These papers overwhelmingly focus on applications in hospital or inpatient settings, with a noticeable gap of applications to single psychology clinics, which this paper aims to add to.

Of the literature studied, Kim et al. (2013) [9] was the only one to employ DES as a tool for improving a mental health clinic. Their objective was to reduce the number of patients seen outside of clinic time, while improving the service times too. Their model successfully replicated the real-world system, and they found that increasing service hours by two and employing an extra psychiatrist resulted in the greatest improvements.

In the literature, DES was also used by Baia Medeiros et al. (2019) [10] to evaluate the impact increases in service demand would have on the ED of an academic hospital, and using it found that increasing bed capacity was the most effective way of meeting the demand.

Aringhieri et al. (2018) [11] devised a hybrid simulation model, combining DES with an agent-based model in order to provide a cost-effectiveness analysis on integrating collaborative care for mental health into primary care pathways in Italy. Their work found the integration to be cost-effective, and successful in reducing GP overcrowding. Their work draws similarities to our project, though we focus solely on the use of DES to reduce waiting times in an psychology 
clinic.

This paper aims to add to the field by presenting the application of DES to a single adult psychology clinic and assess staffing configurations, to allow for better capacity planning by the service.

\section{Method}

For this study, it was agreed to consider the Adult Psychology clinic in Newport as the setting of the work, since the health board identified it as having the most consistent data. The clinic operates from $9 \mathrm{am}$ to $5 \mathrm{pm}$ every weekday.

Upon referral to the clinic, patients will receive an assessment to determine which course of treatment best reflects their needs as a patient. Most patients will require Level One Intervention, which corresponds to a course of group therapy for varying lengths depending on the patient's needs. For some patients, this will be all the treatment needed from the psychology service and they will be released back to their GP upon completion.

There are also Level Two Interventions, in which all patients are given a legal requirement to have a Care Coordinator and a personalised care and treatment plan (CTP). Some patients will access this part of the service immediately from assessment, whilst others access it as a follow up to Level One Intervention, where it is felt additional support is needed. Level Two Interventions consist of courses of individual therapy sessions, where patients can receive a more personalised and specialised CTP.

The individual therapy sessions are split into two types, CTP and CTP enhanced (CTP+). Patients identified as CTP are generally those experiencing moderate levels of distress, while those identified as $\mathrm{CTP}+$ experience more complex and severe problems, that require more focused care coordination and a longer course of individual therapy sessions. Upon completion of Level Two Interventions, patients will continue to meet with their Care Coordinator for a period afterwards, before fully exiting the service. A generalised overview of the patient pathways through the service can be viewed in Figure 2 to aid the above description.

The clinic in Newport employs seven full-time and one part-time Practitioner Psychologists, as well as two Assistant Psychologists, that shadow the Practitioner Psychologists in group therapy sessions. Given the differing complexity and severity of the mental illnesses of the patients, each patient is likely to have a considerably different treatment plan to the others. In Level One Interventions, some patients receive a set course of 12 group therapy sessions, while for others this could rise up to 30 . In Level Two, CTP individual therapy courses last for 24 sessions, with an average of 16 hours of Care Coordinator meetings per year, while $\mathrm{CTP}+$ courses last for 52 sessions, with more regular Care Coordinator meetings at an average rate of 4 hours per month. There are also the behavioural traits of certain patients too, in that some are more likely to not attend the initial assessment, or drop out of the service than others.

Given that it is just this one psychology clinic being considered, and considering the heterogeneity of the patients involved, it was decided that a DES model was apt for researching the problem, using Simul8 simulation software. DES gives the advantage of being able to model healthcare systems where there is a considerable amount of variation between each patient that enters the service, and where we are studying the service in a narrow context, and not looking at it's interactions with other healthcare services. Our final model of the simulation can be viewed in Figure 3 .

The building blocks of Simul8 include start points, queues, activities, end points, resources, and work items, as well as routing arrows between these. The start point can be translated in terms of the clinic as the referral into psychology. The assessments, therapy sessions and Care Coordinator referrals can all be represented using activities, and an end point can be used to route out patients from the service. The work items in this simulation are the mental health patients in the clinic, and the resources are the different types of therapists and the dedicated Care Coordinators.

The activities are set with the set times for the length of each session, as well as the resources required to carry out the session. The model too makes an astute use of labelling and distributions, assigning each patient label values set by probability profiles or named distributions, to reflect the stochastic behaviour of different treatment plans and dropout rates from patient to patient. A full list of the input parameters used in the model can be viewed in Table 1, which were created using information and data made available to us by the health board.

Patients are assigned labels which create their treatment pathway through the service. The labels determine whether the patient will need Level One intervention only, Level Two intervention only, or Level One followed up by Level Two, as well as the length of any group therapy courses, the style of any individual therapy pathways (CTP or CTP+), and the number of sessions they will continue to have with their Care Coordinator upon completion of their therapy course. Using labels, the model is also able to track the number of therapy sessions and Care Coordinator meetings each patient has attended, by acting as a counter that iterates 


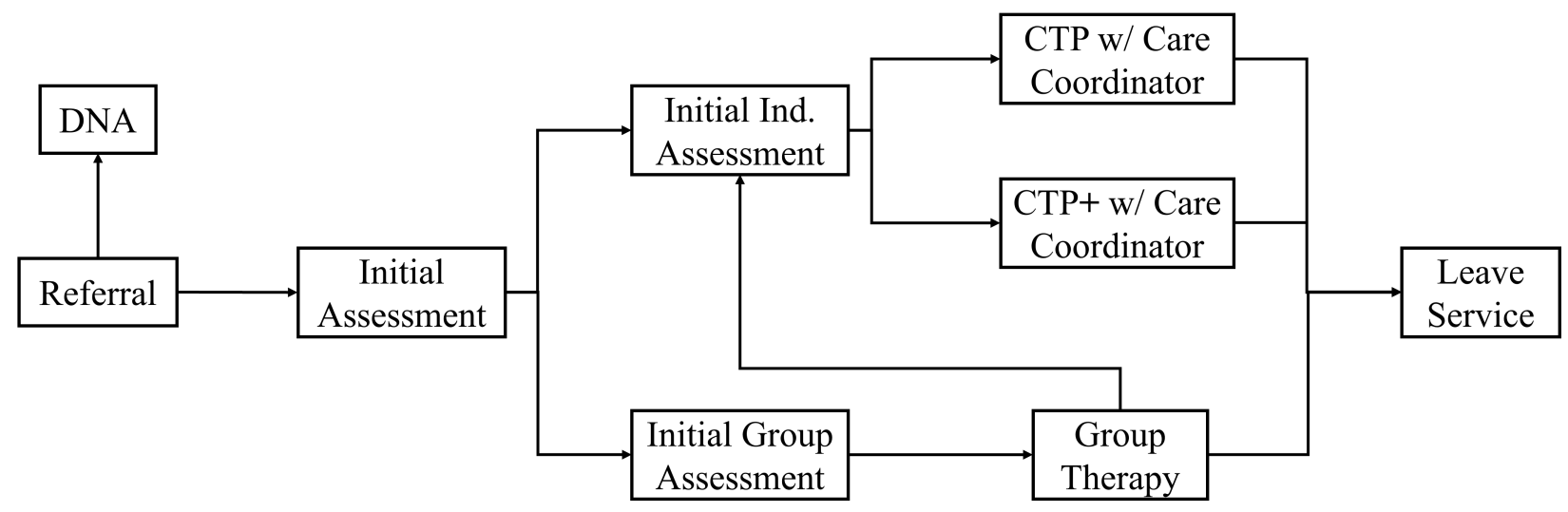

Figure 2. Overview of the patient pathways in the mental health clinic

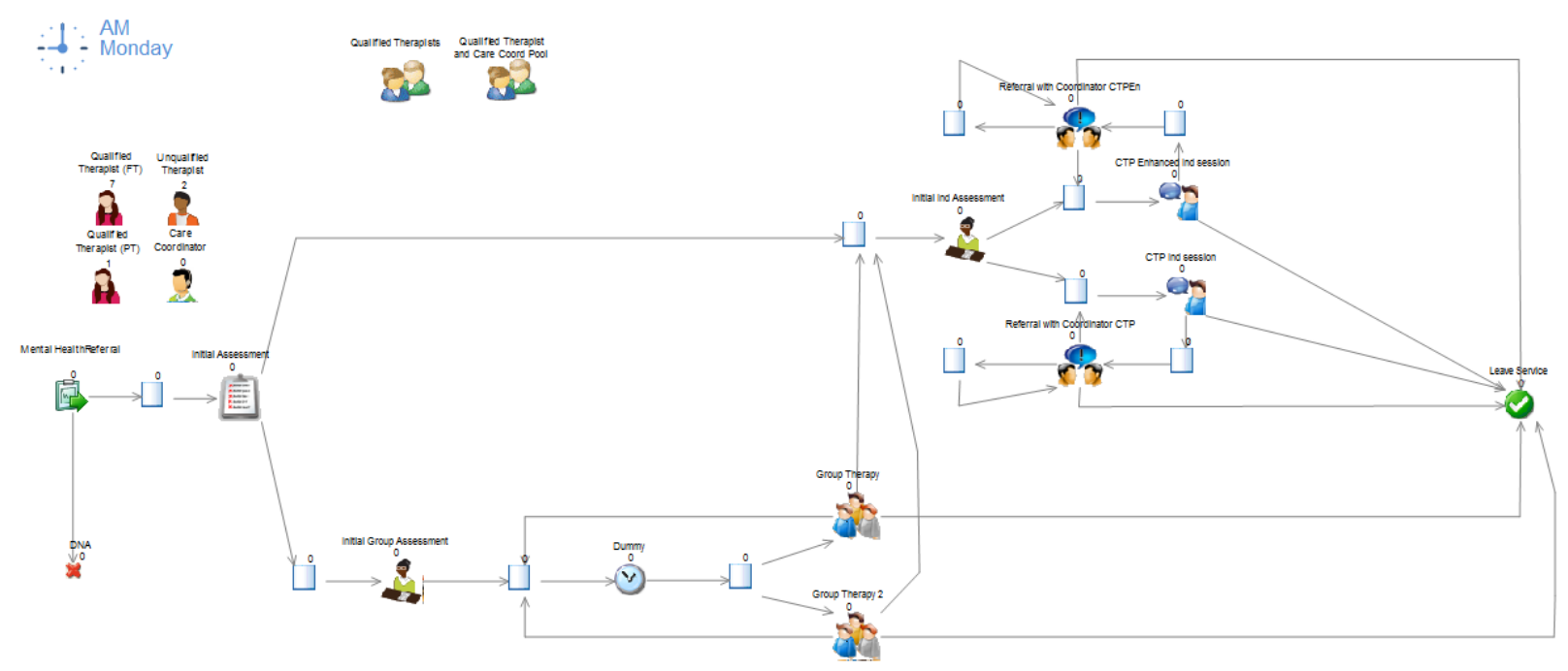

Figure 3. Our DES Simul8 model of the adult psychology clinic in Newport

each time the patient passes through the session. A label is also used as a counter for CTP Care Coordinator meetings. Since these are less frequent than the weekly CTP+ meetings, the label iterates so that the timing distribution for the Care Coordinator meeting is set to 60 minutes for the meeting every 3 weeks, and is 0 otherwise.

After each therapy session, or Care Coordinator meeting, the patient is placed back into the queue for another therapy session. Each queue has a minimum waiting time of one week to simulate the gap between each therapy appointment, and is sorted so that patients that have attended the most sessions are placed at the top of the queue, as a way to replicate a structured weekly appointment timetable. Once the patient has completed their required number of sessions, they are then routed around the model accordingly, in the way that they would progress through the service in the real world.

Resources are also set to take one-hour breaks after each session too, to reflect preparation time for the next sessions, writing up notes for the previous session, as well as lunch and coffee breaks.

The arrival distribution is based on data from the clinic over the 3 month period of November 2019 to January 2020, in which 38 patients were referred to psychology. For the arrival distribution to reflect a degree of seasonality, we took data from StatsWales on the number of referrals to LPCMHSSs in ABUHB from February 2019 to January 2020 [3]. Using the data, we calculated that there were a total of 4873 referrals to LPCMHSSs over the period of November 2019 to January 2020, and calculated that 38 referrals into the psychology clinic accounts for approximately $0.78 \%$ of this figure. This percentage was then used to calculate what the number of referrals to psychology 
Table 1. Overview of the input parameters used in the model

\begin{tabular}{|c|c|c|}
\hline Parameter & Method & Description \\
\hline Arrival rate & Fixed value & Fixed value based on information from ABUHB and StatsWales [3] \\
\hline Therapy times & Fixed values & Fixed values based on information from ABUHB \\
\hline Activity priority & Fixed values & $\begin{array}{l}\text { Higher values give greater priority. Priority determined through } \\
\text { discussion with ABUHB }\end{array}$ \\
\hline $\begin{array}{l}\text { Minimum } \\
\text { queuing times }\end{array}$ & Fixed values & Fixed values used to simulate the wait between therapy sessions \\
\hline Pathway label & $\begin{array}{l}\text { Probability } \\
\text { profile }\end{array}$ & $\begin{array}{l}\text { Assigns patients a value of } 1,2 \text { or } 3 \text { based on a probability profile } \\
\text { to determine which care pathway the patient needs. Distribution } \\
\text { calculated from clinic data }\end{array}$ \\
\hline $\begin{array}{l}\text { Group therapy } \\
\text { type }\end{array}$ & Binary value & $\begin{array}{l}\text { Assigns a binary value based on what length of group therapy the } \\
\text { patient needs. Determined by a probability profile provided by } \\
\text { ABUHB }\end{array}$ \\
\hline $\begin{array}{l}\text { Group therapy } \\
\text { total sessions }\end{array}$ & $\begin{array}{l}\text { Fixed/Triangular } \\
\text { dist. }\end{array}$ & $\begin{array}{l}\text { Depending on whether a short or longer course of group therapy is } \\
\text { required, patients receive a fixed value of 12, or a triangular distribution } \\
\text { calculated with ABUHB }\end{array}$ \\
\hline $\begin{array}{l}\text { Group drop out } \\
\text { rate }\end{array}$ & Binary value & $\begin{array}{l}\text { Assigns a binary value based on whether the patient is likely to drop } \\
\text { out of group therapy }\end{array}$ \\
\hline $\begin{array}{l}\text { Group session } \\
\text { counter }\end{array}$ & Fixed value & $\begin{array}{l}\text { Initial fixed value of } 0 \text {, incremented after completion of a therapy } \\
\text { session for routing purposes }\end{array}$ \\
\hline Ind. therapy type & Binary value & $\begin{array}{l}\text { Assigns a binary value based on if this patient is CTP or CTP+. } \\
\text { Determined by a probability profile provided by ABUHB }\end{array}$ \\
\hline Ind. drop out rate & Binary value & $\begin{array}{l}\text { Assigns a binary value based on whether the patient is likely to drop } \\
\text { out of individual therapy }\end{array}$ \\
\hline $\begin{array}{l}\text { Ind. session } \\
\text { counter }\end{array}$ & Fixed value & $\begin{array}{l}\text { Initial fixed value of } 0 \text {, incremented after completion of a therapy } \\
\text { session for routing purposes }\end{array}$ \\
\hline Care Coord. & Binary value & $\begin{array}{l}\text { Assigns a binary value based on whether the patient's care coordination } \\
\text { is handled by the clinic }\end{array}$ \\
\hline $\begin{array}{l}\text { Care Coord. total } \\
\text { sessions }\end{array}$ & Triangular dist. & $\begin{array}{l}\text { Unique triangular distributions for both CTP and CTP+ patients to } \\
\text { determine the number of individual sessions they will have }\end{array}$ \\
\hline $\begin{array}{l}\text { Care Coord. } \\
\text { session counter }\end{array}$ & Fixed value & $\begin{array}{l}\text { Initial fixed value of } 0 \text {, incremented after completion of a Care } \\
\text { Coordinator session for routing purposes }\end{array}$ \\
\hline $\begin{array}{l}\text { Care Coord. } \\
\text { session length }\end{array}$ & $\begin{array}{l}\text { Probability } \\
\text { profile }\end{array}$ & $\begin{array}{l}\text { Determined by a probability profile based on whether the patient } \\
\text { receives care coordination by the clinic, or if they do or do not have } \\
\text { a session that week. Time is set to } 0 \text { if no session, } 60 \text { otherwise }\end{array}$ \\
\hline $\begin{array}{l}\text { Care Coord. wait } \\
\text { counter }\end{array}$ & Fixed value & $\begin{array}{l}\text { CTP patients only. Initial fixed value of } 0 \text {, incremented after passing } \\
\text { through the care coordinator activity to highlight a week passing. } \\
\text { Resets to } 0 \text { once it hits } 3 \text { to indicate the patient is due a session }\end{array}$ \\
\hline
\end{tabular}

could potentially have been over the February 2019 to October 2019 period.

Each implementation into the model was carefully tried and tested to ensure that it had the desired effect on the model, and as such, verify that it runs in a way that captures the patient flow in the real-world system. In order to do so, aspects of the simulation were extracted from the model, and run in their own smaller sub-simulation, with endpoints placed in all areas routing out of the sub-simulation, to check that the correct percentage of patients were being routed to the correct areas of the simulation. Additionally, these added endpoints were sometimes replaced with queues. In doing so all patients that would exit the sub-simulation are routed into queues that have no routing out destination. Doing so allowed the contents of the queues to be checked, to verify that labels were incriminating and being assigned as they should and were also routing the patients around the service as intended. 


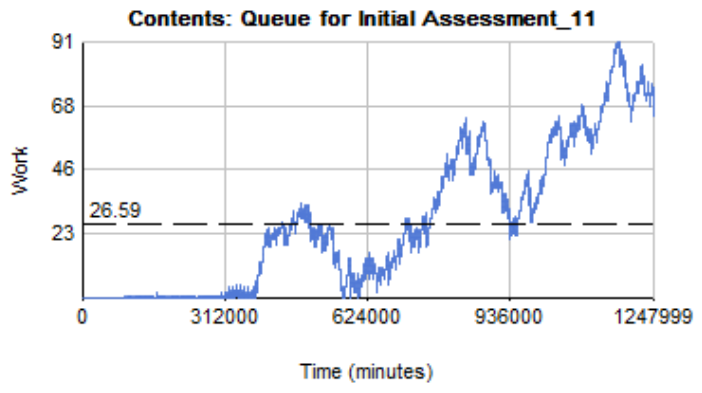

Figure 4. Model results of the number of people waiting for an initial assessment over a $\mathbf{1 0}$ year period, from one iteration (Graph is in simulation time)

\section{Results}

The most important aspects of the model to consider in the results collection are the waiting times for the activities, and perhaps most importantly of all, the waiting times for the initial assessment activity, as that was identified by the health board as the key area where waiting times had to be reduced.

To prime the model for results collection, we ran the model with the therapists as the Care Coordinators several times over a period of 10 years and paid close attention to how the number of patients waiting for the initial assessment developed over that period. Doing so allowed a better understanding of how patient flow was affected over time and allowed identification of a suitable warm-up period for the simulation.

We elected to pursue a warm-up period of 3 years. Figure 4 presents the the number of patients waiting for an initial assessment over the 10 year period. As seen by the figure, it is at approximately 3 years (in simulation time) where we can see noticeable changes to the number of people waiting, with a sustained bottleneck for over a year afterwards, providing us with the closest time period to a steady state in the system. Hence, it was a useful point to start assessing the effect of employing dedicated Care Coordinators and as such, the results collection period was run for 1 year after the warm-up period. There was no information from the health board on people leaving the service after waiting for so long, therefore queue baulking was not included in the model, which could have provided a steady state period.

Using Simul8's trial calculator, the software calculated that for this model, approximately 2000 runs per trial would be needed to obtain a precision of 5 percent in this model for the most important result, the average waiting time for the initial assessment. One trial was then run for each scenario, considering different staffing configurations of the service.

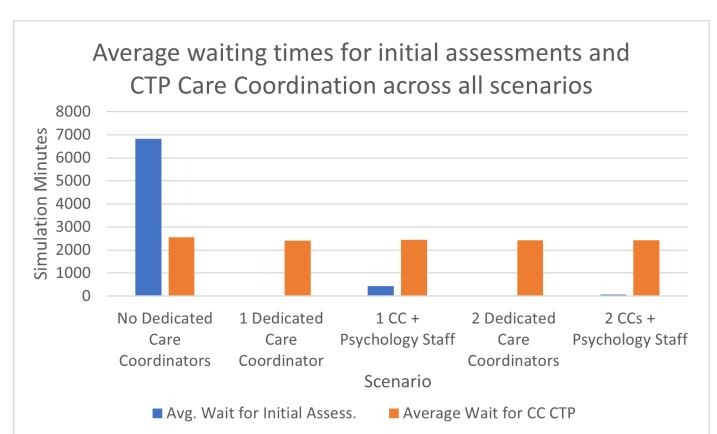

Figure 5. Trial results for average waiting times for initial assessments and CTP Care Coordination across all scenarios

The first scenario was to test how the system performs based on its current operation in the real world, where the qualified therapists act as the Care Coordinators alongside their other roles. Scenarios were also run where they were replaced as the Care Coordinators by at first one dedicated Care Coordinator, and then two dedicated Care Coordinators. Additionally, scenarios were run whereby the psychologists shared the coordination role with one dedicated Care Coordinator, and then shared with two dedicated Care Coordinators.

It was found that the service in its current configuration, where the psychologists must undertake the Care Coordination roles, performs sub-optimally across all metrics. It quite considerably had the highest average waiting time for initial assessments out of all configurations by over 6000 minutes, as seen in Figure 5. Given the assumptions and limitations in this model this is likely to be much higher in the real system. The current configuration also has slightly inflated average waiting times for individual therapy assessments, group therapy assessments, and CTP Care Coordinator referrals. This is owing largely to the over-utilisation of the therapists at the clinic, who must prioritise seeing CTP+ patients alongside the other aspects of the clinic.

Where dedicated Care Coordinators were employed, the average wait times were cut significantly in all configurations, performing best where there was just the one or two dedicated Coordinators undertaking the roles. Employing these Care Coordinators enabled the other staff to focus on their other roles in the clinic, allowing for an increased number of assessments undertaken in those configurations. The exception to this is for the scenario where just one Care Coordinator was employed, however. Despite performing excellently across all other metrics, this configuration performed poorest in the number of completed Care Coordinator referrals, and in fact, was unable to perform a single 
referral for CTP patients, as viewed in Figure 6. This was shown by the Coordinator having a utilisation rate of $100 \%$, and with such a high level of utilisation, they were constantly focused on the prioritised CTP+ patients which lead to neglect of the CTP patients.

Depending on the financial resources available to the clinic, the best options to take might be the blended approach of employing one, or two, Care Coordinators to undertake the roles alongside the therapists. Despite not performing the best among some metrics such as the average waiting times for initial assessments and starting therapy courses, the trials still produced strong results, as well as significant improvement on the current configuration. Where the hybrid approach performed best though was in the total number of patients receiving Care Coordination and initial assessments. Especially for the Care Coordination, with the total number of referrals being decisively the highest for both CTP and CTP+ therapy courses. This highlights that these configurations were able to allow the therapists to assess patients entering the service in a more timely and efficient manner, while also having the added benefit of improved Care Coordination with the dedicated staff taking on the bulk of these roles, thus helping to clear backlogs in the service.

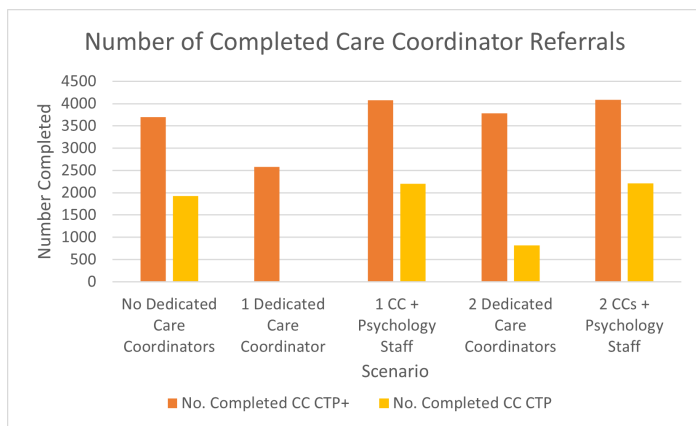

Figure 6. Trial results for total number of completed Care Coordinator referrals across all five scenarios

\section{Limitations and Discussion}

This simulation model is not without its limitations and assumptions. A great deal of effort was sought during the project to try and ensure involvement of the clinic in the development of the model, but even so, some information about the service was unable to be ascertained before the deadline of this report. As such, the model applies several assumptions where necessary to try and capture the patient flow of the service to the best of our understanding. Several assumptions are likely to potentially increase patient flow through the service and hence are not accountable for the real-world clinic. As such, if data on these assumptions was obtained, we would likely see patient flow decrease, and as a result potentially making the waiting times more consistent with the real-world system.

Resources in the model are assumed to have $100 \%$ efficiency in their tasks, and take hour long breaks every hour, or upon completion of a therapy session. Information on this was not obtained from the health board, so the time spent working on other tasks in the clinic was modelled based on discussion with mental health workers. There is also no provision for staff taking sickness days or holidays through the model. Similar too for the patients, aside from the patients with dropout rates, there is no provision for patients who may cancel or postpone their therapy session, hence extending time in the service. Information on the occurrence patterns of this could result in it being implemented into the model with relative ease using labels and timing distributions.

Another limitation was the lack of information on the number of patients in the backlog for the initial assessment, as well as the average waiting times of those waiting for their assessment. Without this data, we were unable to validate the model against the real-world information. This data would provide a target to aim at in the validations process to ensure the model captures the patient flow of the clinic. That said, the model passed each stage of verification, so if data on such was provided, it should be attainable to adjust the model so that it meets these figures.

Fundamentally, the model should be able to be adjusted, or at the least could be used a framework, in modelling the patient pathways of other psychology clinics in ABUHB, NHS Wales and other health services in the United Kingdom. Effort was made to ensure that the model is visually simplified so that it can be understood by workers in these services who perhaps do not have experience with the software, while at the same time, is robust in operation so that it can capture the patient flow in the service.

The results of the model will be used by the health board to influence their service model design in indicating whether the employment of dedicated Care Coordinators is considered worthwhile. It will support management in helping to make a difference to patient waiting times, and coincidentally, in improving patient results.

There is room too for the model to be expanded to consider the clinic's interactions with other aspects of Primary Care and mental health services such as hospital wards, community teams and GP surgeries. The model in this report has the potential to be combined alongside an SD model, as part of a hybrid simulation, to model 
the complex nature of the wider or perhaps whole mental health service network in the geographic location.

\section{Conclusion}

An adult psychology clinic in the United Kingdom was modelled successfully using Discrete Event Simulation. Through input from the project stakeholder as to how the service operates, the model was successfully built and verified based on the information gathered. Our simulation framework provides reasonable assumptions and allows us to capture patient flow in the service. Furthermore, it identifies the bottleneck occurring before the patients' initial assessments.

Using the simulation, results were obtained that presented a strong indication in favour of employing dedicated Care Coordinators to take over the roles from the therapists, additionally highlighting how the current configuration of the service performed in comparison. It seems likely that employing dedicated Coordinators to share the role with the psychologists may be the most effective approach, with the dedicated staff taking on most of the roles to allow the rest of the psychologists to focus on their roles in the rest of the clinic.

In conclusion, DES has proved a powerful tool in modelling a mental health service, evidenced by this model's reflection of the collaborating adult psychology clinic. The findings of this work indicate that simulation can be a useful methodology in improving the accessibility and efficiencies of a psychology clinic, highlighted by the potential improvements shown in the staffing configuration scenarios carried out. With applications of simulation in mental health settings growing, we would urge further research and projects to be undertaken in this field, as it is evidently clear that it can improve these services for the better and provide people with the timely access to mental health treatment that they need.

\section{References}

[1] B. D. Bradley, T. Jung, A. Tandon-Verma, B. Khoury, T. C. Chan, and Y.-L. Cheng, "Operations research in global health: a scoping review with a focus on the themes of health equity and impact," Health research policy and systems, vol. 15, no. 1, pp. 1-24, 2017.

[2] W. Government, "Health status, illnesses, and other conditions (welsh health survey): 2015," 2015.

[3] StatsWales, "Referrals for a LPMHSS assessment, by LHB, age and month," 2021.

[4] R. C. of Psychiatrists, "Census 2019: Workforce figures for consultant psychiatrists, specialty doctor psychiatrists and physician associates in mental health," 2019.
[5] A. Rais and A. Viana, "Operations research in healthcare: a survey," International transactions in operational research, vol. 18, no. 1, pp. 1-31, 2011.

[6] S. C. Brailsford, P. R. Harper, B. Patel, and M. Pitt, "An analysis of the academic literature on simulation and modelling in health care," Journal of simulation, vol. 3, no. 3, pp. 130-140, 2009.

[7] K. M. Long and G. N. Meadows, "Simulation modelling in mental health: A systematic review," Journal of Simulation, vol. 12, no. 1, pp. 76-85, 2018.

[8] S. Noorain, K. Kotiadis, and M. P. Scaparra, "Application of discrete-event simulation for planning and operations issues in mental healthcare," in 2019 Winter Simulation Conference (WSC), pp. 1184-1195, IEEE, 2019.

[9] B. Kim, Y. Elstein, B. Shiner, R. Konrad, A. S. Pomerantz, and B. V. Watts, "Use of discrete event simulation to improve a mental health clinic," General hospital psychiatry, vol. 35, no. 6, pp. 668-670, 2013.

[10] D. T. Baia Medeiros, S. Hahn-Goldberg, D. M. Aleman, and E. O'Connor, "Planning capacity for mental health and addiction services in the emergency department: a discrete-event simulation approach," Journal of healthcare engineering, vol. 2019, 2019.

[11] R. Aringhieri, D. Duma, and F. Polacchi, "Integrating mental health into a primary care system: A hybrid simulation model," in New Trends in Emerging Complex Real Life Problems, pp. 55-63, Springer, 2018. 